\title{
On the vortical structure in a round jet
}

\author{
Tadashi Matsuda ${ }^{\text {a) }}$ and Jun Sakakibara \\ Department of Engineering Mechanics and Energy, University of Tsukuba, \\ Tennodai 1-1-1, Tsukuba 305-8573, Japan
}

(Received 26 March 2004; accepted 3 November 2004; published online 24 January 2005)

\begin{abstract}
Turbulent vortical structures in a round free jet of water were experimentally visualized by using stereo particle image velocimetry (PIV). A laser light sheet illuminated a cross-sectional plane normal to the axis of the jet, and two charge-coupled device cameras captured particle images in the same region of interest but from different directions. The stereo-PIV algorithm had been applied to obtain two-dimensional, three-component (2D-3C) velocity distributions on various cross-sectional planes along the axis downstream. All nine components of the velocity gradient tensor were reconstructed from time-dependent $2 \mathrm{D}-3 \mathrm{C}$ velocity data by locally assuming Taylor's frozen field hypothesis based on the convective velocity evaluated from the mean flow profile. Isosurfaces of the swirling strength $\lambda_{i}$ revealed that the existence of a group of hairpinlike vortex structures was quite evident around the rim of the shear layer of the jet. The center of curvature of the head of the hairpin was typically observed around $r / b=1.5$, and the azimuthal spacing between the legs of the hairpin was roughly $0.9 b$. A similar hairpin structure was estimated by linear stochastic estimation. The typical spacing between the legs of the estimated hairpin was $0.65 b$, which is generally constant over the range of $\mathrm{Re}=1500-5000$. (C) 2005 American Institute of Physics.
\end{abstract}

[DOI: $10.1063 / 1.1840869]$

\section{INTRODUCTION}

The present research is concerned with the spatial structure in the far-field (or self-preserving) region of an incompressible turbulent round jet. A round free jet has been well investigated both analytically and experimentally. ${ }^{1,2} \mathrm{Al}-$ though the existence of large-scale structures in this region has been recognized by flow visualization such as laserinduced fluorescence (LIF), ${ }^{3}$ there have still been many difficulties in extracting the geometrical detail of the coherent structure due to the temporal and spatial uncertainty of its occurrence.

The organized structure in the far-field region is much less axisymmetric, while that in the near-field region is explained as an apparently axisymmetric mixing layer dominated by vortex rings generated by the Kelvin-Helmholtz instability. ${ }^{4,5}$ It is supposed that a vortex ring in the near-field grows disturbed as the flow goes downstream and is transformed to a gathering of many pieces of a particular vortex structure, which consists of a pair of radial flows outward and inward with respect to the jet. ${ }^{6}$ The scale of these partial structures is comparable to the local width of the jet. ${ }^{7}$

Tso and Hussain ${ }^{8}$ measured the velocity field in the farfield region of a round jet by employing an array of several hot-wire probes. They tried to determine the chief spatial modes of the vortex structure from the velocity correlation. They found three major modes-axisymmetric, helical, and double helical-and concluded the helical mode was the most dominant. A LIF visualization by Dimotakis $e t$ al. ${ }^{3}$ also led to a hypothesis that indicated the existence of axisymmetric and helical structures and their transitional structure.

\footnotetext{
${ }^{a)}$ Present address: Toshiba Corp., 1-1-1, Shibaura, Minato-ku, Tokyo 1058001, Japan.
}

On the contrary, Yoda $^{7}$ disagreed with the large-scale helical structure and instead suggested a sinusoidal structure from her LIF experiments. The helical structure was also doubted by Ninomiya, ${ }^{9}$ who extracted the organized structure by applying linear stochastic estimation to the velocity field, which was obtained by three-dimensional PTV (par-

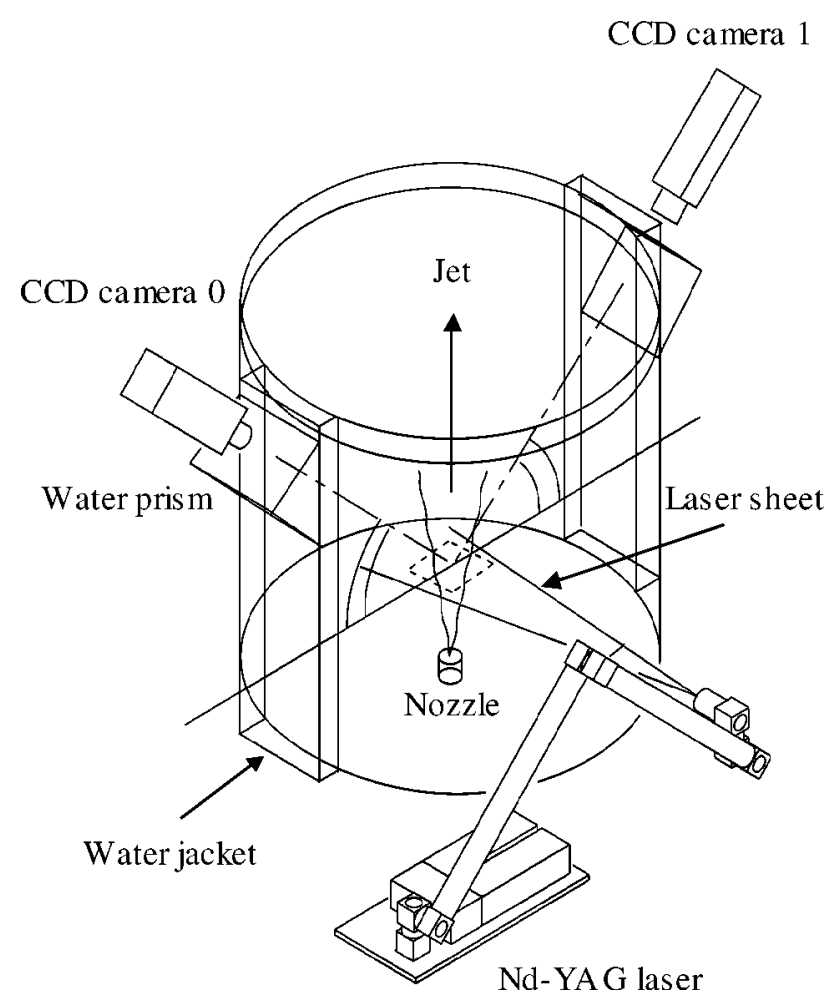

FIG. 1. Schematic of stereo-PIV facility. 


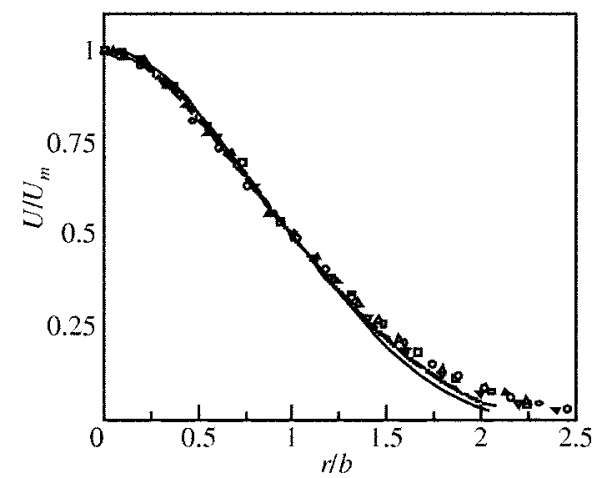

(a) Mean streamwise velocity

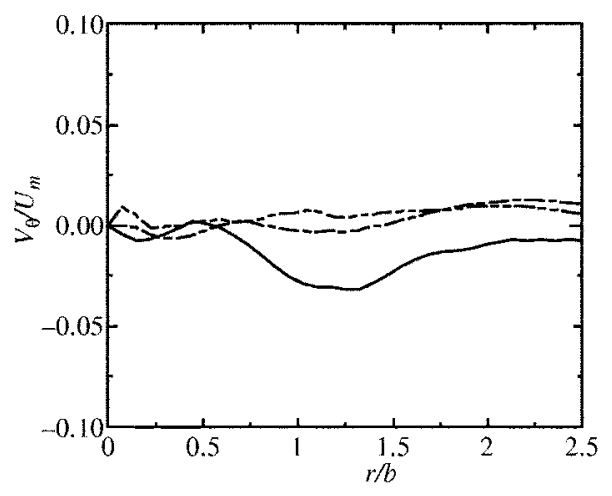

(c) Mean azimuthal velocity profile

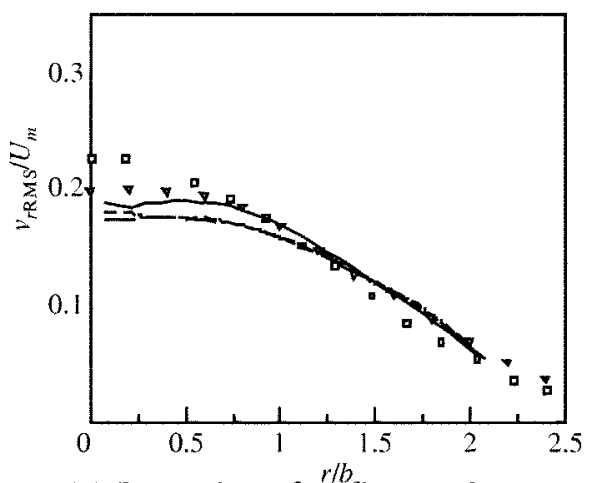

(e) Intensity of $v_{\mathrm{r}}^{r / b}$ fluctuation across the jet.

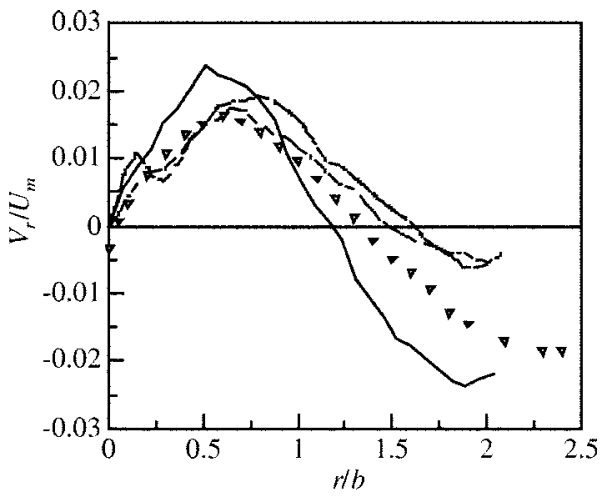

(b) Mean radial velocity plofile.

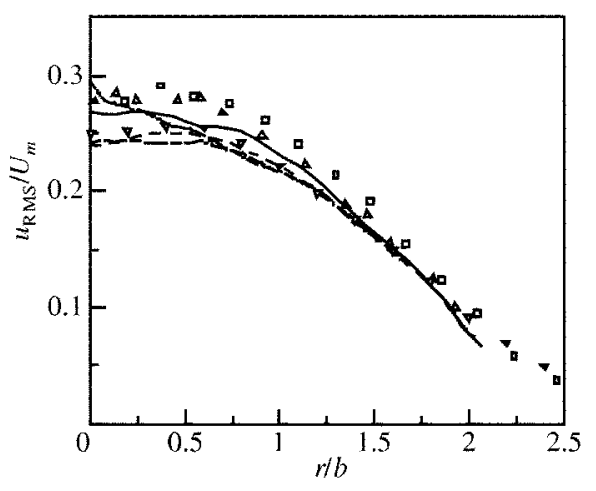

(d) Intensity of $u$ fluctuation across

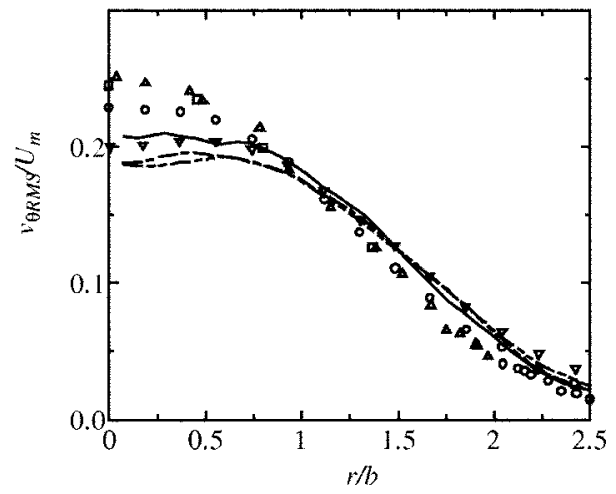

(f) Intensity of $v_{\theta}$ fluctuation across

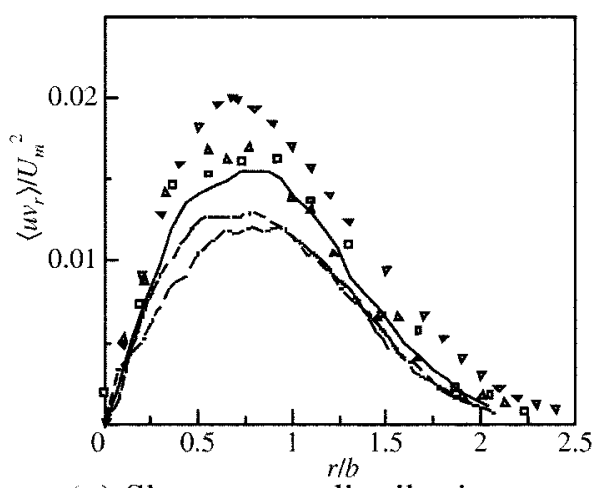

(g) Shear stress distribution across

FIG. 2. Statistical quantities of the jet. —, Re=1500; —-—, Re=3000; —.-—, Re=5000; $\bigcirc, \triangle, \square,---$, Wygnanski and Fiedler (1969); $\nabla$, Ninomiya (1992). 


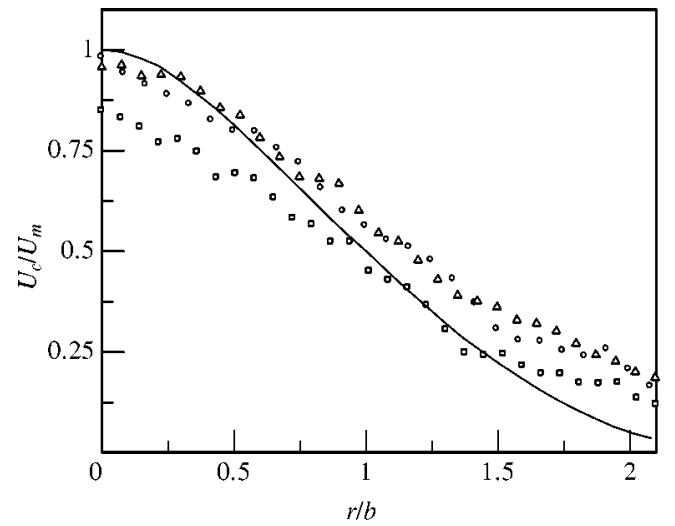

(a) Convective velocity normalized by maximum mean streamwise velocity.

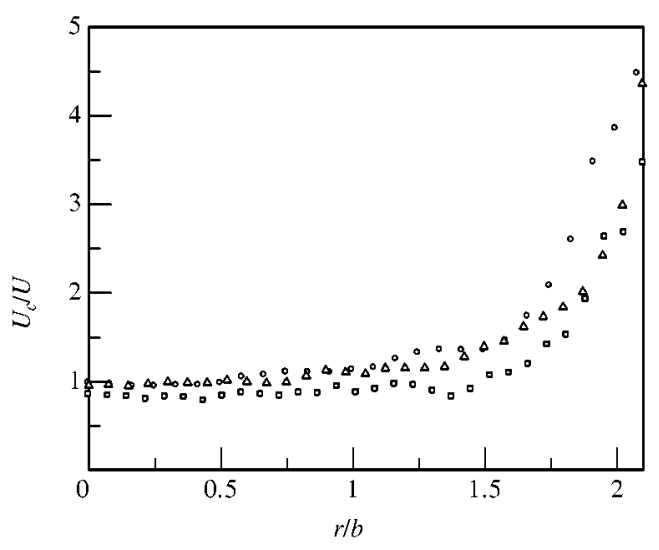

(b) Ratio of convective velocity to local mean velocity

FIG. 3. Convective velocity at $x=30 D$ (a) and ratio of convective velocity to local mean velocity (b). - , mean velocity profile at $\mathrm{Re}=3000 ; \mathrm{O}, \mathrm{Re}$ $=1500 ; \triangle \operatorname{Re}=3000 ; \square, \operatorname{Re}=5000$.

ticle tracking velocimetry). A recent numerical study suggests the existence of a group of hairpin-shaped vortices inclined downstream, ${ }^{10}$ which might explain the characteristics of the statistical properties reported in earlier research.

Our interest was to learn the actual instantaneous vortex distribution and the organized structure extracted from the velocity field around the jet. Using stereo PIV, we first measured the velocity fields over the axial cross section of the far-field region of a round free jet of water at several Reynolds numbers, on the order of $10^{3}$ based on the nozzle diameter, and visualized a quasi-three-dimensional velocity field, assuming Taylor's frozen field hypothesis. Next, we performed a linear stochastic estimation to extract the typical geometrical distribution of the structure.

\section{EXPERIMENTAL EQUIPMENT AND PROCEDURE}

The flow was a round turbulent water jet emerging from a nozzle (diameter $D=5 \mathrm{~mm}$ ) with Reynolds numbers of 1500-5000. Water was driven from a reservoir tank out the nozzle at a steady velocity with a top-hat profile into a cylindrical Plexiglas tank (1040 mm high; $780 \mathrm{~mm}$ diameter). The water was seeded homogeneously with polyamide-12 (a)

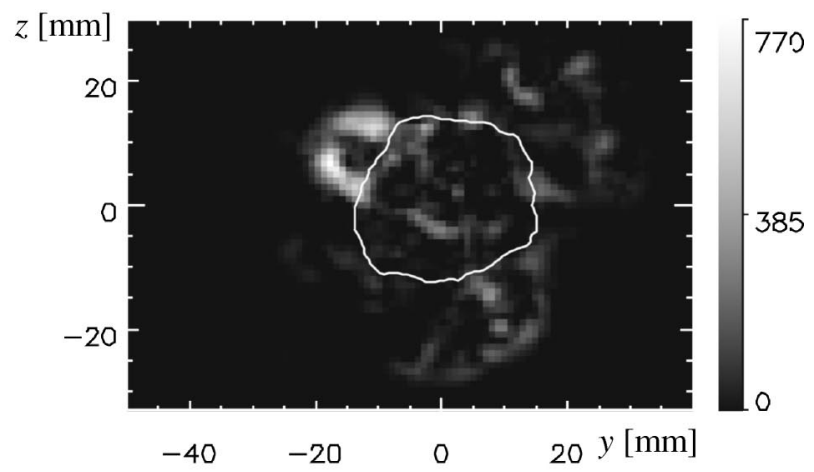

(b)

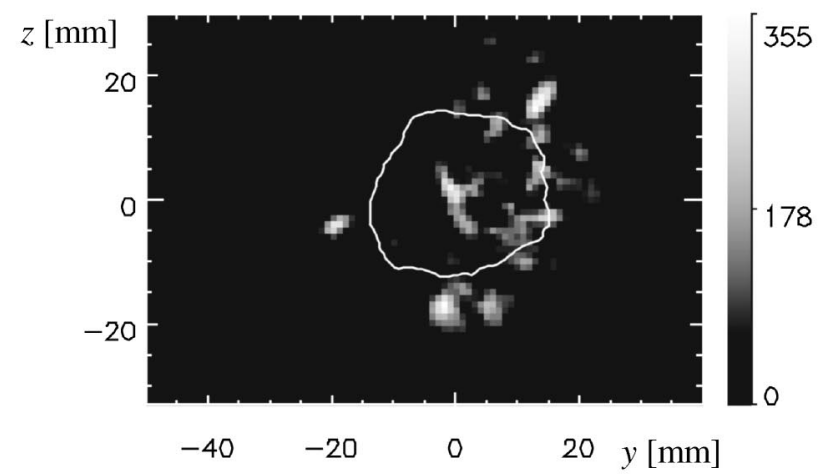

FIG. 4. Intensity distributions of $\lambda_{i}$ across the jet at two different instances (a) and (b).

tracer particles (Daicel-Degussa, DAIAMID: 1.02-1.03 sp gr, $40 \mu \mathrm{m}$ average diameter). The nozzle was placed in the center of the bottom of the cylindrical tank, followed by a section with a flow straightener and a perforated plate below the exit (Fig. 1).

We employed a stereoscopic PIV that is capable of resolving time-dependent, three-component velocity in the planes $20 D-50 D$ downstream of the nozzle. These regions of interest were well beyond the minimum far-field criterion ${ }^{4}$ of $x / D \geqslant 20$. A laser light sheet of $2 \mathrm{~mm}$ in thickness was produced by a Nd:YAG laser (New Wave Research, Minilase-II/30 Hz) through a cylindrical lens illuminated planes normal to the streamwise direction, and two chargecoupled device (CCD) cameras (Kodak, Megaplus ES1.0) were positioned to view the tracer particles at the same region of interest in the light sheet plane. The angle between the axes of the two cameras was set to about $90^{\circ}$, and their lenses were mounted to satisfy the Scheimpflug condition. The images captured by the CCD cameras were stored in the host memory of a PC through image grabbers (Imaging Technology, IC/PCI) with a frame rate of $30 \mathrm{fps}$. Our PIV system could typically capture 450 successive timedependent pairs of images for both cameras. Since a velocity field could be measured based on the double images, 225 instantaneous three-component velocity maps with $1 / 15 \mathrm{~s}$ intervals were obtained for every run of the experiment.

The time interval separating the two PIV single exposures was set in order to adjust the mean displacement of the 


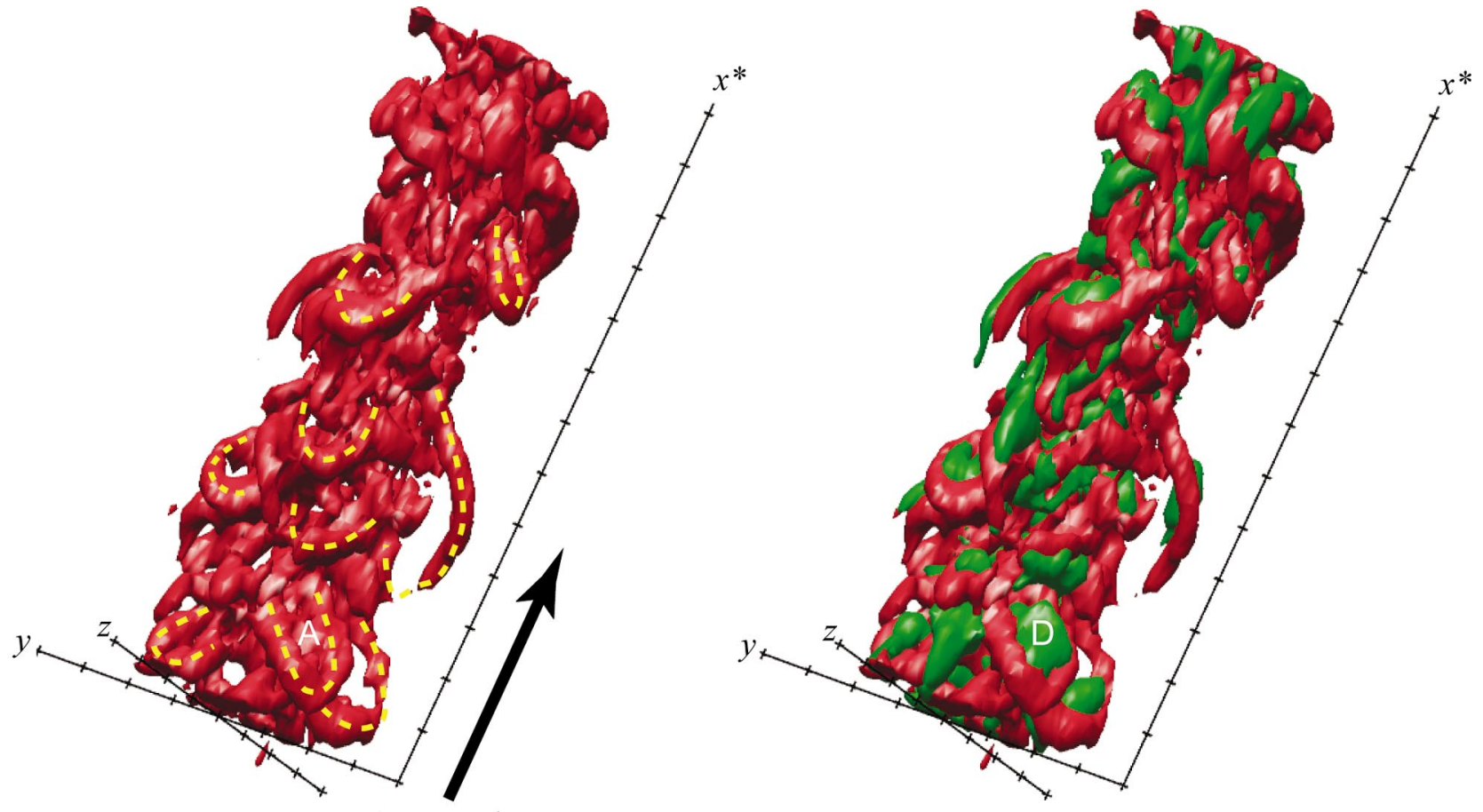

(a)

Streamwise

(c)

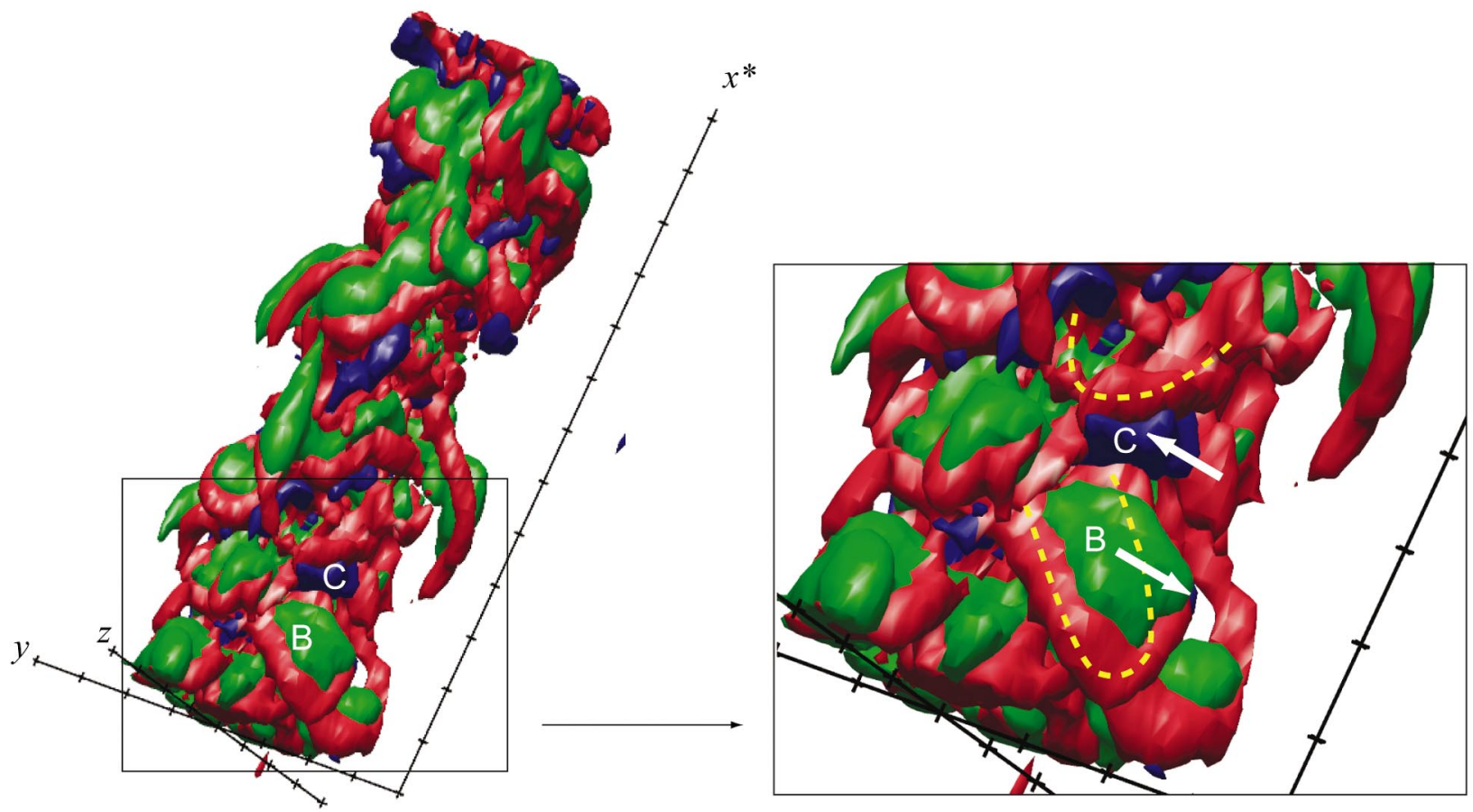

(b)

FIG. 5. (Color). Vortex strings and their involved flow at $x=20 D, \operatorname{Re}=3000$, displayed as isosurfaces of $\lambda_{i}=0.6 b / U_{m}$. The tick interval of the $x^{*}, y$, and $z$ axes is $b$. The yellow dotted lines on (a) indicate the appearance of vortex strings. (b) is overlayed with the isosurface of $\widetilde{v}_{r}=0.1 U_{m}$ (green) and $\widetilde{v}_{r}=-0.1 U_{m}$ (blue), and (b) also shows a closeup of the hairpin vortex. (c) is overlayed with the isosurface of $u v_{r}=0.015 U_{m}{ }^{2}$.

particle at the center line of the jet to $\approx 10$ pixels. Since the error in measuring the displacement of the tracers was within 0.2 pixels, the error of the instantaneous velocity was estimated to be $2 \%$ of the maximum mean velocity.
The spatial resolution of the velocity measurement was limited by the size of the interrogation spot. Consider a sinusoidal velocity with spatial wavelength $L$ and size $N$ of the interrogation spot of the PIV. Gain $G$ of the measured veloc- 
(a) $\operatorname{Re}=1500$
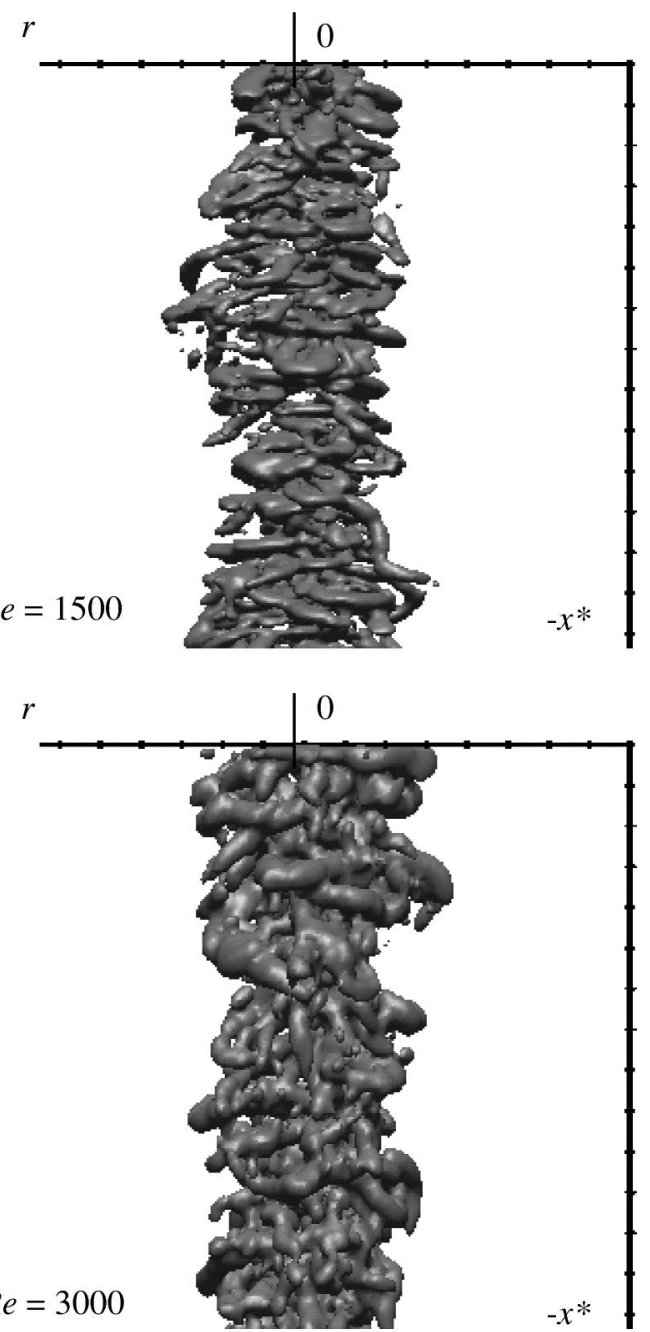

(b) $R e=3000$

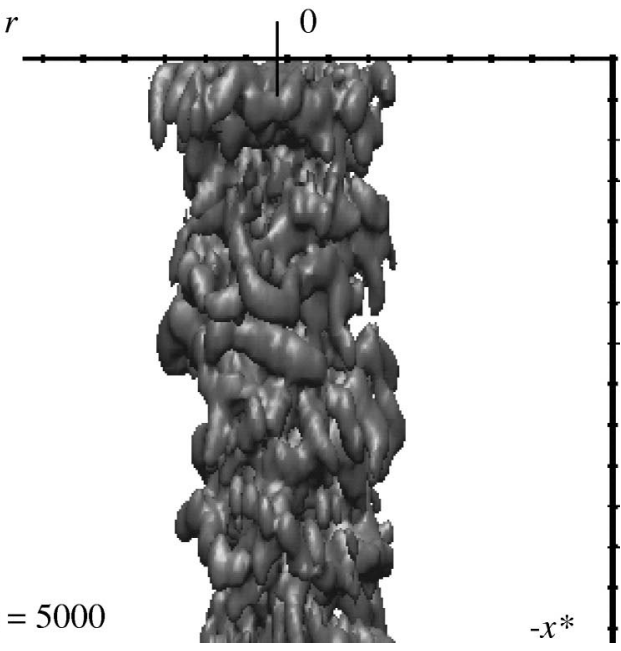

(c) $R e=5000$

FIG. 6. Isosurfaces of $\lambda_{i}$ at $\operatorname{Re}=1500,3000,5000, x=30 D$. The tick interval is $b$.

ity compared with the true velocity was derived according to the following equation used by Hart: ${ }^{11}$

$$
G=\left|\frac{\sin (\pi N / L)}{\pi N / L}\right| .
$$

In the present measurements, the dimensions of the interrogation spot were approximately $N_{z}=2.6 \mathrm{~mm}$ (28 pixels) in

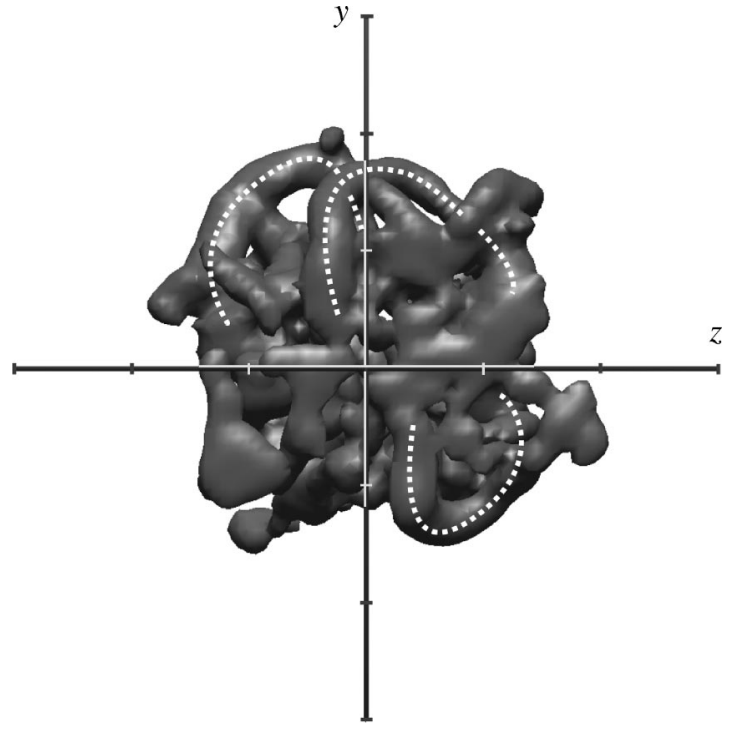

FIG. 7. Vortex strings from the viewpoint on the jet axis, displayed as isosurfaces of $\lambda_{i}$ at $x=30 D, \operatorname{Re}=3000$. White dotted lines indicate the appearance of vortex strings. The tick interval is $b$.

the $z$ direction and $N_{y}=1.9 \mathrm{~mm}$ ( 28 pixels) in the $y$ direction. Thus, the wavelength at which the gain dropped 50\% $(G$ $=0.5)$ was computed as $4.3 \mathrm{~mm}$ and $3.2 \mathrm{~mm}$ in the $z$ and $y$ directions, respectively. This wavelength was $\approx 17 \%-29 \%$ of the local jet half-width, and 30-120 times larger than the Kolmogorov scale of the present flow. Obviously, the results shown in this paper will be focused on large-scale structures without resolving small dissipative eddies.

We employed a rectangular Cartesian coordinate system $(x, y, z)$, where $x$ is the vertical (streamwise) distance from the nozzle exit, and $y$ and $z$ are the axes of the plane normal to $x$. In addition, this coordinate system must be transformed into a three-dimensional polar coordinate system $(x, r, \theta)$ for subsequent processes. We employed the least-squares method on the two-dimensional profile of the streamwise component of the velocity to detect the point where the jet axis penetrates the measurement plane, which was set at the origin, $r=0$. The mean velocities for the streamwise, radial and azimuthal components are $U, V_{r}$, and $V_{\theta}$, respectively, and $u, v_{r}$, and $v_{\theta}$ are the velocity fluctuations of each component.

To visualize (or to reconstruct) the three-dimensional vortex from the continuous sets of velocity fields obtained, the following assumption was needed. Our stereo-PIV system could resolve the velocity distribution only on a twodimensional plane; nevertheless, the axis normal to the plane could be locally defined as $x^{*}=-U_{c} t$ if we assumed Taylor's frozen field hypothesis, which states that the flow structure remains unchanged as it passes downstream, or, formally, that $\partial / \partial t=-U \partial / \partial x$ for a homogeneous flow. Here $U_{c}$ is the convective velocity of a spatial structure and $U$ is the local mean velocity. Although a round turbulent jet is not rigorously homogeneous in the $x$ direction, this hypothesis is still valid as long as it is used with a convective velocity of appropriate scale. $^{2}$

The distribution of vortices was visualized by employing 


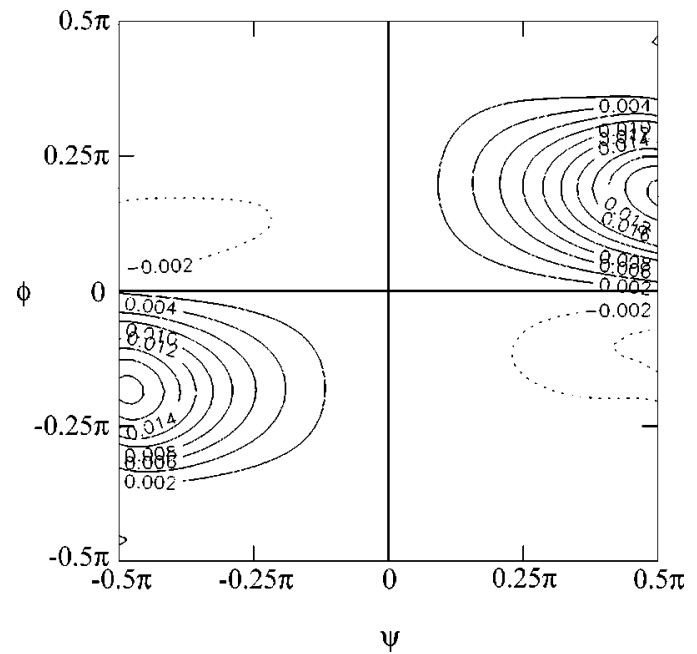

FIG. 8. Joint probability density function of the angle of the velocity fluctuation vectors weighted by $u v_{r}$ for $\mathrm{Re}=3000$ measured at all possible azimuthal locations centered at $(x, r)=(30 D, 1.0 b)$.

the swirling strength $\lambda_{i}$, which is a more appropriate index than vorticity, since $\lambda_{i}$ represents only the intensity of the rotating fluid motion and does not involve the contribution of shearing transformation. ${ }^{12,13} \lambda_{i}$ corresponds physically to the angular velocity of the local swirling motion and mathematically to the imaginary part of the complex eigenvalue pair of the local velocity gradient tensor. The local velocity field around a particular point at radius $\mathbf{r}$ is expressed as

$$
\mathbf{u}(\mathbf{r}+\delta \mathbf{r})=\mathbf{u}(\mathbf{r})+\mathbf{A} \delta \mathbf{r}+O\left[(\delta \mathbf{r})^{2}\right],
$$

where $\mathbf{A}$ is the velocity gradient tensor. Then, we get $\lambda_{i}$ as the imaginary part of the eigenvalue $\lambda$ by solving the characteristic equation,

$$
\operatorname{det}(\mathbf{A}-\lambda \mathbf{I})=0 \Leftrightarrow \lambda^{3}+P \lambda^{2}+Q \lambda+R=0,
$$

where the constant $Q$ is known to be an index often employed in the identification of vortices. A comparison between the structures visualized by vorticity and $\lambda_{i}$ is given in Appendix A.

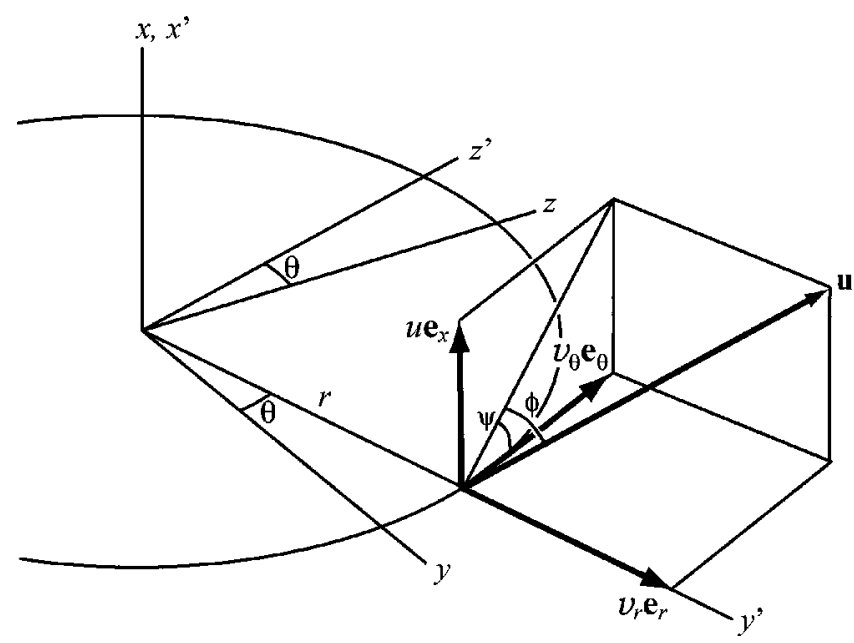

FIG. 9. Definition of angle of velocity vectors $\phi$ and $\psi$. Unit vectors $\mathbf{e}_{x}, \mathbf{e}_{r}$, and $\mathbf{e}_{\theta}$ are in the $x^{\prime}, r$, and $\theta$ directions, respectively.

\section{SINGLE-POINT STATISTICS}

The mean and rms velocity profiles are shown in Fig. 2. The radius is normalized by $b$, which is the local half-value width of $U$, and the velocities are normalized by $U_{m}$, which is the maximum value of $U$. These profiles are obtained by an ensemble average over 450 stereo-PIV realizations for each condition. Most of these statistical quantities show good agreement with previous research, such as Wygnanski and Fiedler $^{2}$ or Ninomiya. ${ }^{9}$

The data for the streamwise mean velocity, shown in Fig. 2(a), collapses nicely into the plots of Wygnanski and Fiedler. ${ }^{2}$ The radial velocity in Fig. 2(b) also shows reasonable agreement with Ninomiya's PTV study, ${ }^{9}$ although $V_{r}$ is fairly low in magnitude, such as $2 \%$ of the mean streamwise velocity. The negative values of $V_{r}$ observed on the outer side of the jet appear to be the entrainment; the flow inward from the surrounding fluid. The mean azimuthal velocity for the $\mathrm{Re}=3000$ and $\mathrm{Re}=5000$ cases, shown in Fig. 2(c), deviates $0.014 U_{m}$ from zero at the maximum, while that for the $\operatorname{Re}=1500$ case shows a slightly larger deviation $\left(0.032 U_{m}\right)$. The flow in the tank might have some large-scale rotation around the jet axis, although care was taken to ensure the fluid in the tank was quiescent before the experiment. The intensity of the velocity fluctuation in Figs. 2(d)-2(f) also agrees well with previous work.

Figure 2(g) shows the Reynolds shear stress $\left\langle u v_{r}\right\rangle$ normalized by $U_{m}{ }^{2}$, where \langle\rangle denotes the streamwise average and the ensemble average. The greatest exchange of momentum, which is supposed to involve large-scale vortices, is found to occur at $r \leqslant 2 b$.

\section{VISUALIZATION OF VORTICES}

Although it is important to estimate the convective velocity of turbulence $U_{c}$ when we assume Taylor's frozen field hypothesis to reconstruct an imaginary three-dimensional velocity field, the assumption that $U_{c}$ is constant for the entire measurement plane across the jet is hardly valid. ${ }^{2}$ The convective velocity in the present jet was measured in the following way. Two-dimensional, two-component velocity maps on the plane including the jet axis were first measured by regular two-component PIV. Then the cross correlation of the streamwise component of the velocity at two points separated by $\delta x$ in the streamwise direction and separated by constant time $\Delta t$ in the temporal direction was calculated. The convective velocity $U_{c}$ was determined by $U_{c}$ $=\delta x_{\max } / \Delta t$, where $\delta x_{\max }$ is the $\delta x$ giving the maximum cross correlation. The profile of measured convective velocity $U_{c}$ is shown in Fig. 3. The convective velocity is slightly lower than $U$ on the jet axis but increases with radius $r$ to become more than $U$. The $U_{c}$ at a higher Reynolds number tends to be lower.

Nevertheless, it is still worth using the limited frozen hypothesis to transform temporal quantities to spatial quantities in a range of $\Delta r$ such that the convective velocity can be considered nearly constant. This statement might sound paradoxical, but it is reasonable as long as we only observe the characteristic organized structure located around a certain radius. In order to ascertain where the large-scale vortices are 

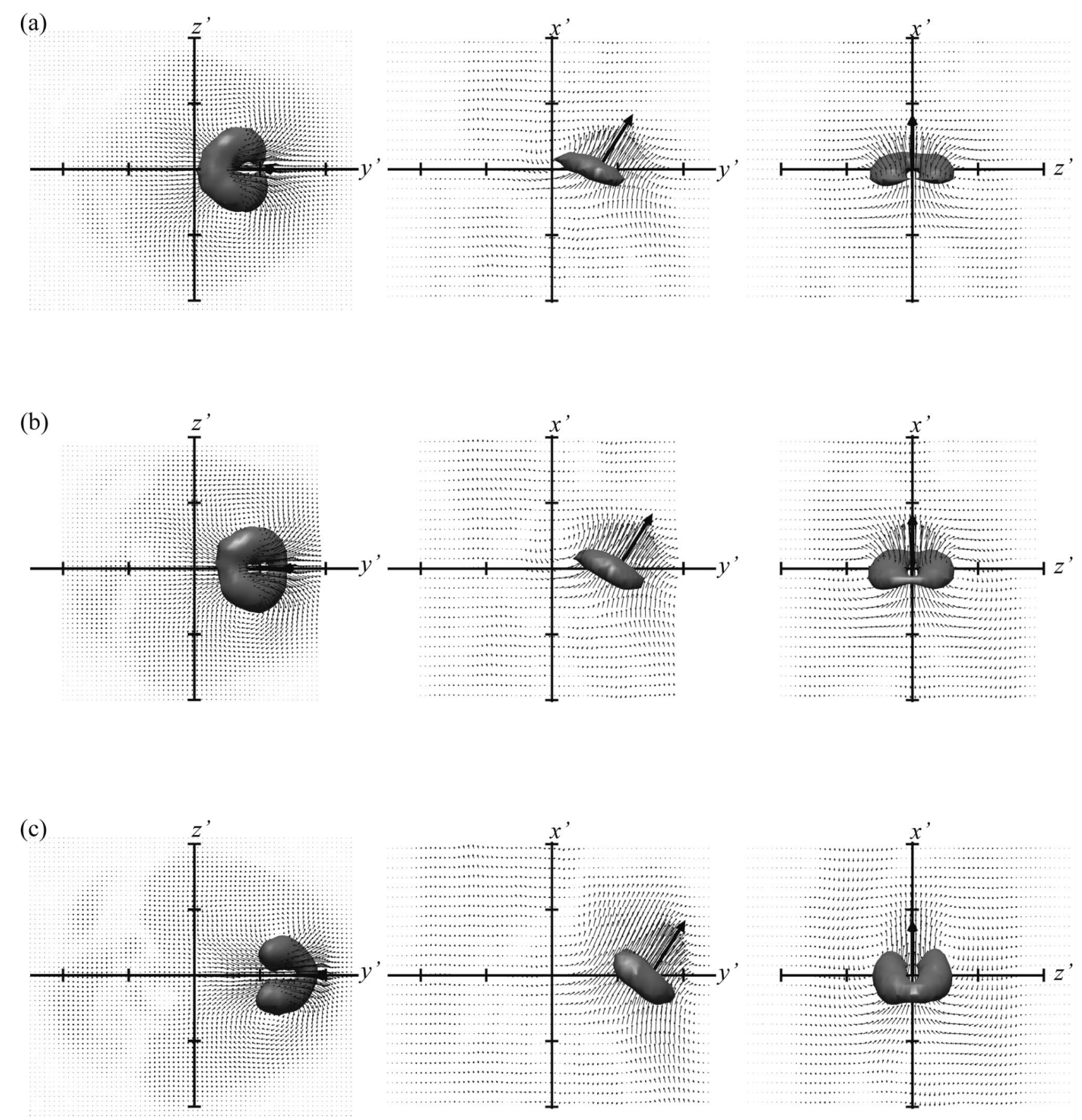

FIG. 10. Estimated conditional velocity vectors and those isosurfaces of $\lambda_{i}=1.1|\mathbf{u}(\mathbf{x})| b^{-1}$ at $x=30 D, \operatorname{Re}=3000$. The tick interval is $1.0 b$. The thick arrows represent the event vector $\mathbf{u}(\mathbf{x})$. The reference points are (a) $0.7 b$, (b) $1.0 b$, and (c) $1.5 b$, respectively. Three different views for each case are shown from left to right.

located on the normal cross section of the jet, we tentatively tried a $\lambda_{i}$ visualization with a certain $U_{c}$. Figure 4 shows two examples of the intensity distribution of $\lambda_{i}$ with $U_{c}=0.3 U_{m}$ in a section normal to the jet axis. The shaded gauges on the right side of the diagrams represent the intensity of $\lambda_{i}$ using an arbitrary unit of measurement. The white contour indicates the half value of the axial mean velocity $U$, which has a mean radius of $b$. Although it still needs to be confirmed whether these diagrams can rigorously be called the spatial distribution of swirling intensity, since the calculation itself is based on the assumption of uniform convective velocity, we can still observe the uneven distribution of $\lambda_{i}$, which forms structures comparable in size to the jet width.

In Fig. 4, some large "lumps" of $\lambda_{i}$ appear beyond $b$ and near the outer rim of the jet. Near width $b$, the radial gradient of $U_{c}$ is significant and causes difficulty in visualizing the structure striding over $b$ without any distortion. On the other hand, near the outer region of the jet, where the gradient of $U_{c}$ is relatively small, vortex structures could show their reconstructed spatial characteristics, no matter how partially.

Based on the discussion above, we visualized isosurfaces of $\lambda_{i}$ over the imaginary three-dimensional space transformed from the successive time-dependent sets of the twodimensional velocity fields obtained by stereo PIV. In this 


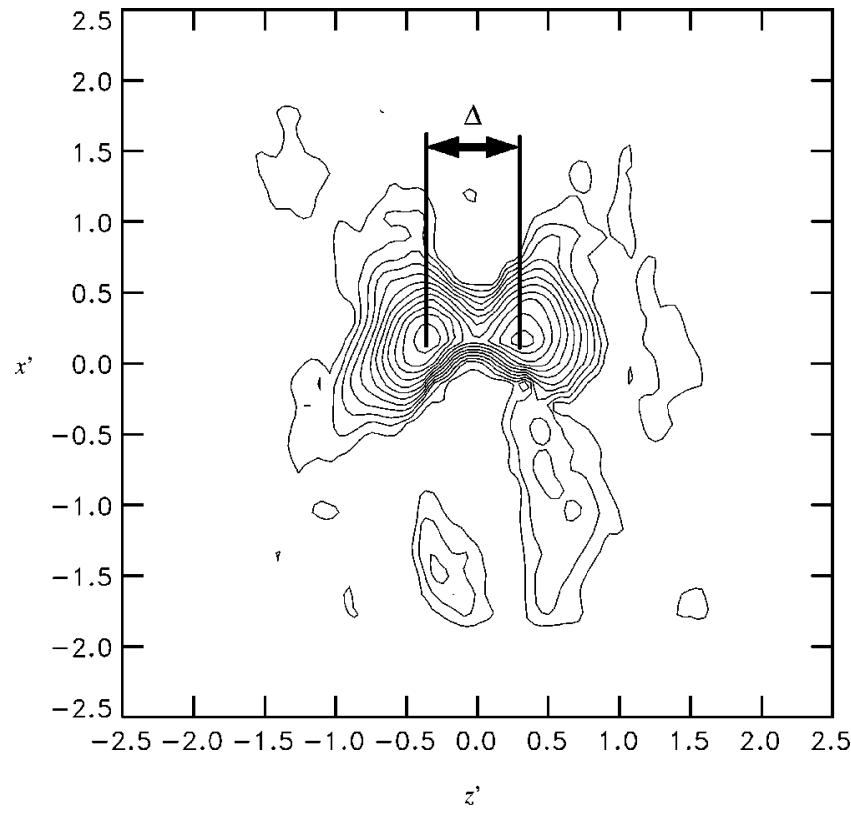

FIG. 11. Contours of $\lambda_{i}$ of the estimated conditional velocity vectors in the $x^{\prime}-z^{\prime}$ plane at $y^{\prime}=1.0 b$ for the reference point $y^{\prime}=1.5 b$. The Reynolds number is $\operatorname{Re}=3000$, and the streamwise location where the raw velocity data was taken is $x=30 D$.

case, the convective velocity $U_{c}$ was assumed to be $U_{c}$ $=\alpha U_{m}$ with a constant coefficient $\alpha=0.3$. The value of the coefficient $\alpha(=0.3)$ is determined empirically (or expediently) in the process of attempting $\lambda_{i}$ visualization, but it is also reasonable to represent the convective velocity with $r$ $\approx 1.5 b-2.0 b$, according to the profile of $U_{c}$. Note that the effect of the choice of $\alpha$ on the visualized structures is given in Appendix B.

Figure 5(a) shows isosurfaces of $\lambda_{i}$ at $x=20 D$, Re $=3000$, where the streamwise coordinate ( $x^{*}$ coordinate) is defined by the measurement time $t$ as $x^{*}$ $=-U_{c} t$. At first glance, the hairpin structures, emphasized by the yellow dotted lines, are observed hanging upstream or twining around the entire jet. The $\lambda_{i}$ spots found in Fig. 4 are assumed to be cross sections of these hairpin structures. In order to know the physical size of the hairpin, we traced the vortex strings, which resemble the hairpins empirically (by visual inspection), and measured the width and streamwise distance between successive hairpins. The width, which was defined as the distance between hairpin legs at a relative streamwise location $0.8 b$ downstream from the head of the hairpin, was $0.9 b$, and the streamwise distance was $1.8 b$ on average, although this value may depend strongly on the method of identification of the hairpin. Note that structures similar to the present hairpin were also observed in the LES studies of the round free jet performed by Suto et al. ${ }^{10} \mathrm{Al}-$ though it is difficult to abstract a particular structure from the visualization at this moment, we can barely discover helical structures or vortex rings comparable in size to the jet width from any visualization we made. Figure $5(\mathrm{~b})$ shows isosurfaces of the radial component of velocity $\widetilde{v}_{r}$ together with that of $\lambda_{i}$, where the green and blue surfaces indicate positive and negative values, respectively. It is obvious that $\lambda_{i}$ can represent the swirling intensity with the radial flows injecting

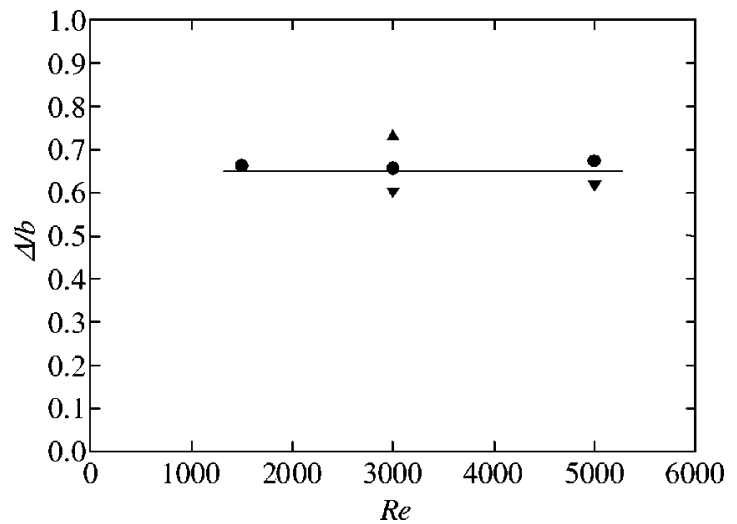

FIG. 12. Spacing $\Delta$ of the legs of the hairpin defined as the distance between two maximums of $\lambda_{i}$ in the $x^{\prime}-z^{\prime}$ plane at $y^{\prime}=1.0 b$. The reference point was $y^{\prime}=1.5 b$. $\nabla, x / D=20 ; \bullet, x / D=30 ; \boldsymbol{\Delta}, x / D=40$.

outward (e.g., label $B$ ) through the inside of the hairpin [e.g., label $A$ of Fig. 5(a) $]$ and entrained to the rim of the structures (e.g., label $C$ ). As shown in Fig. 5(c), such an injecting flow induced by the hairpin vortex transfers the streamwise momentum outward, which indicates isosurfaces of the instantaneous Reynolds shear stress $u v_{r}$. Many of the isosurfaces of $u v_{r}$ are located inside the hairpin loop (e.g., label $D$ ), where the flow induced by the hairpin vortices transfers the high streamwise momentum in the core region of the jet toward the outer region. The probability density function of the direction of the velocity fluctuation vector weighted by the Reynolds stress will be discussed in Sec. V.

Figure 6 shows the visualized isosurface of $\lambda_{i}$ at $x$ $=30 \mathrm{D}$. The scale of the radius is $1.0 \mathrm{~b}$. Let us observe the visualizations according to the Reynolds number. The vortices look shrunken at the low Reynolds number (1500) and extended at the high Reynolds number (5000) in the axial direction. This shows that $U_{c}$ was estimated as larger at the high Reynolds number and smaller at the low Reynolds number. The fall of $U_{c} / U_{m}$ with the increase in the Reynolds number, which we could also ascertain in Fig. 3, supports this fact. Therefore, it is difficult to impartially discuss the distribution of vortices, but still the visual azimuthal wavelength of the isosurface does not look very different at any Reynolds number. Note that because the visual azimuthal wavelength $(\approx 0.9 b)$ is much larger than the spatial resolution $(<0.29 b)$ mentioned previously, the observation is not limited by the spatial resolution of the measurement. A more objective measure of the size of the hairpin vortices will be discussed in the following section.

Figure 7 shows vortices from the viewpoint on the jet axis at $x=30 D, \operatorname{Re}=3000$. Here, the center of curvature of the head of hairpin is found to appear roughly at $r=1.5 \mathrm{~b}$, and the azimuthal range of the angle occupied by a hairpin leg seems less than $90^{\circ}$.

\section{LINEAR STOCHASTIC ESTIMATION}

In order to extract the geometrical characteristics of the vortex structure more objectively, we applied linear stochastic estimation ${ }^{14}$ to the velocity field. The quantity of interest is the linear estimate of the conditional average of the veloc- 
ity field based on an event consisting of the velocity at a single point. The conditional average of the $i$ th component of the velocity vector at position $\mathbf{x}^{\prime}$ based on the $j$ th component of the event velocity vector at reference position $\mathbf{x}$ is written as $\left\langle u_{i}\left(\mathbf{x}^{\prime}\right) \mid u_{j}(\mathbf{x})\right\rangle$. The equations for linear stochastic estimations of the $i$ th component of $\left\langle u_{i}\left(\mathbf{x}^{\prime}\right) \mid u_{j}(\mathbf{x})\right\rangle$ are

$$
\begin{aligned}
\hat{u}_{i}\left(\mathbf{x}^{\prime}, \mathbf{x}\right) & =\text { linear estimate of }\left\langle u_{i}\left(\mathbf{x}^{\prime}\right) \mid u_{j}(\mathbf{x})\right\rangle \\
& =\sum_{j=1}^{3} L_{i j}\left(\mathbf{x}^{\prime}, \mathbf{x}\right) u_{j}(\mathbf{x}) .
\end{aligned}
$$

The manipulation for minimizing the mean square error of the estimate leads to linear algebraic equations for the estimation coefficients $L_{i j}$ :

$$
\sum_{j=1}^{3}\left\langle u_{j}(\mathbf{x}) u_{k}(\mathbf{x})\right\rangle L_{i j}\left(\mathbf{x}^{\prime}, \mathbf{x}\right)=\left\langle u_{k}(\mathbf{x}) u_{i}\left(\mathbf{x}^{\prime}\right)\right\rangle, \quad j, k=1,2,3 .
$$

Once $L_{i j}$ is obtained, the flow field $\hat{u}_{i}$, called "conditional eddies," ${ }^{14}$ is given by the event vector. In the present study, the event vector corresponding to the value that contributes the largest amount of the mean Reynolds shear stress was chosen, as previously performed by Ninomiya. ${ }^{9}$ A joint probability density function of the angle of the velocity fluctuation vectors weighted by $u v_{r}$, referred to as WPDF, measured at all possible azimuthal locations centered at $(x, r)$ $=(30 D, 1.0 b)$ is shown in Fig. 8. Here, the angles of the vectors are defined as

$$
\psi=\tan ^{-1}\left(u /\left|v_{\theta}\right|\right) \text { and } \phi=\tan ^{-1}\left|v_{r} /\left(u^{2}+v_{\theta}^{2}\right)^{1 / 2}\right|,
$$

which are illustrated in Fig. 9 in conjunction with a rotating rectangular coordinate $\left(x^{\prime}, y^{\prime}, z^{\prime}\right)$. The Reynolds number for this case was $\operatorname{Re}=3000$. There are two distinct peaks at $(\phi, \psi)=(-0.48 \pi,-0.18 \pi)$ and $(0.50 \pi, 0.18 \pi)$, and each corresponds to inward $\left(v_{r}<0\right)$ and outward $\left(v_{r}>0\right)$ motions, respectively. Since these peaks represent the fluctuating velocity vector which makes the most contribution to the Reynolds shear stress, we defined the event vector as the unit vector, which has the angle $(\phi, \psi)$ where the positive peak is located in the WPDF. Here, only the event vector corresponding to the peak in $\phi>0$, which represents outward motion, was used in the following.

The estimated conditional velocity vectors and isosurfaces of $\lambda_{i}=1.1|\mathbf{u}(\mathbf{x})| b^{-1}$ at $x=30 D, \operatorname{Re}=3000$ are shown in Fig. 10. Here, results for three different reference points are indicated, and a thick arrow represents the event vector. The surfaces for the reference point at $y^{\prime}=0.7 b$, where the mean Reynolds stress reaches a maximum, exhibit a $C$-shaped vortex inclined with respect to the $y^{\prime}$ axis, as shown in Fig. 10(a). While the region inside the reference point $\left(y^{\prime}\right.$ $<0.7 b)$ has a thick surface of $\lambda_{i}$ extending around the reference point, the $\lambda_{i}$ isosurface outside the reference point $\left(z^{\prime}\right.$ $=0$ and $\left.y^{\prime}>0.7 b\right)$ is discontinued. In the case of the reference point $y^{\prime}=1.0 b$ shown in Fig. 10(b), the surface of $\lambda_{i}$ outside the reference point $\left(y^{\prime}>1.0 b\right)$ is connected, but is thinner than the other part, and forms a ringlike shape. Such a structure was also found in the linear stochastic estimation performed by Ninomiya. ${ }^{9}$
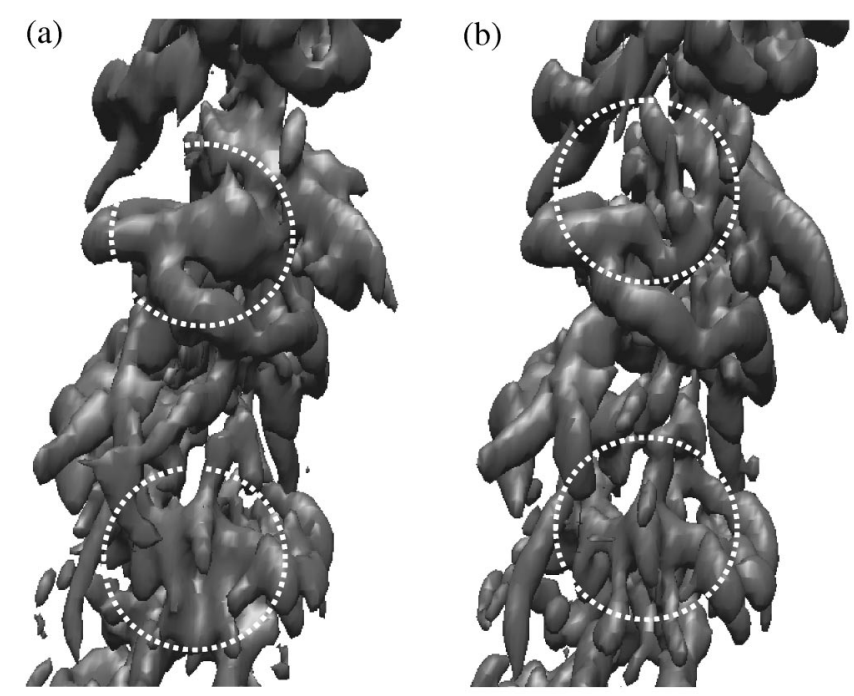

FIG. 13. Visualized vortices, displayed as isosurfaces of vorticity (a) and $\lambda_{i}$ (b) at $x=30 D, \operatorname{Re}=3000$.

The structure estimated for the reference point at $y^{\prime}$ $=1.5 b$, shown in Fig. 10(c), exhibits a more pronounced feature: the inside of the reference point $\left(z^{\prime}=0\right.$ and $\left.y^{\prime}<1.5 b\right)$ is completely disconnected and the outside of the reference point $\left(z^{\prime}=0\right.$ and $\left.y^{\prime}>1.5 b\right)$ is connected by a thick surface, and forms a $U$-shaped hairpinlike vortex. Since the reference point $\left(y^{\prime}=1.5 b\right)$ is located at the typical center of curvature of the hairpin's head visualized in the preceding section, the structure estimated here stochastically supports the existence of the hairpin vortices.

The spacing $\Delta$ of the legs of the hairpin is defined as the distance between the two maximums of $\lambda_{i}$ in the $x^{\prime}-z^{\prime}$ plane at $y^{\prime}=1.0 b$. The contour map of $\lambda_{i}$ in the $x^{\prime}-z^{\prime}$ plane at $y^{\prime}$ $=1.0 b$ for the reference point $y^{\prime}=1.5 b$ is shown in Fig. 11 . There are two distinct peaks, which correspond to the legs of the hairpin shown in Fig. 10(c). The distance between these peaks $\Delta$ was measured for each case and plotted in Fig. 12 . For all cases in the range of $\mathrm{Re}=1500-5000$, the $\Delta$ is $\approx 0.65 b$. Note that Nickels and Marusic ${ }^{15}$ proposed a simple structural model of the round jet, which consists of the hairpin-type vortices arranged to extend the head toward the radial direction and the legs toward the center line. The azimuthal spacing of their proposed hairpin was $\approx 0.51 b$ at the leg and $0.17 b$ at the head.

\section{CONCLUDING REMARKS}

Stereo-PIV measurement was applied to the selfpreserving region of a round free water jet at $\mathrm{Re}$ $=1500-5000$. The statistical properties of turbulence obtained in this measurement agreed well with previous analytical and experimental research. Assuming that $U_{c}$ is locally uniform near the vortex structure around the outer rim of a jet, we reconstructed the quasi-instantaneous threedimensional velocity field from the successive timedependent data of three components of the velocity field on the axial cross section of a jet obtained by stereo PIV. The 
(a)

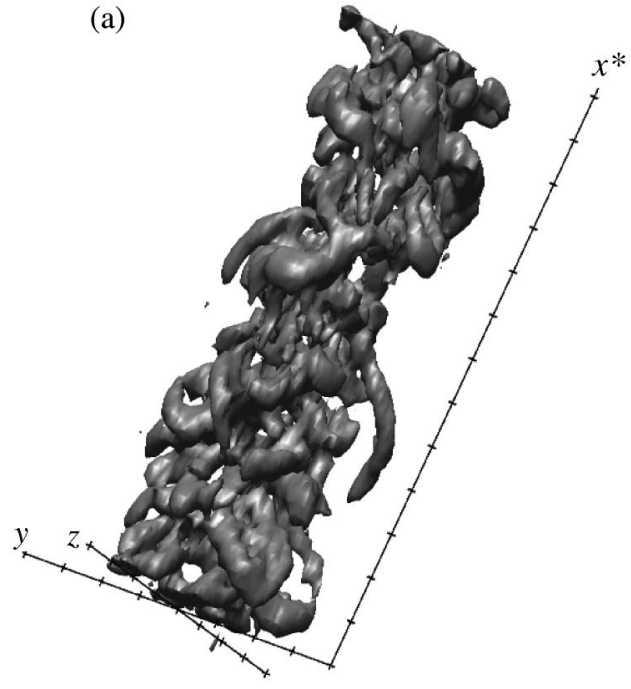

(b)

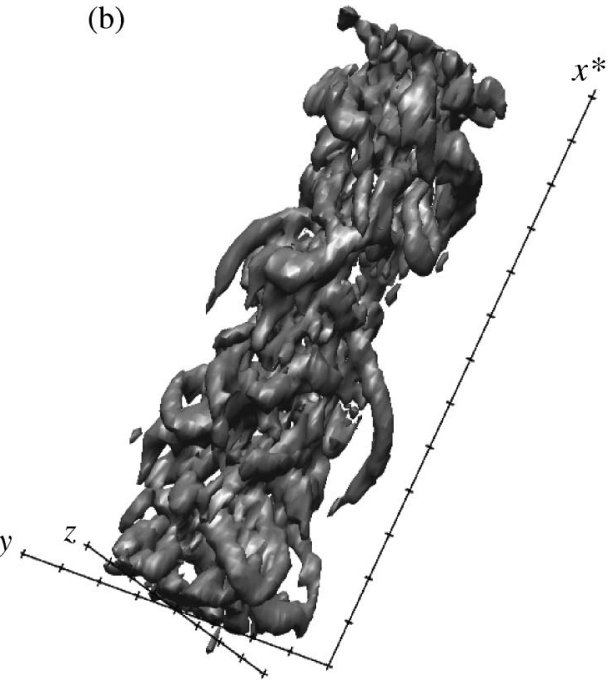

FIG. 14. Visualized vortices, displayed as isosurfaces of $\lambda_{i}$ at $x=20 D$, $\mathrm{Re}=3000$ with use of convective velocity $U_{c}=0.2 U_{m}$ (a) and $U_{c}=0.5 U_{m}$ (b). vortex structures in the far-field region of the round jet were visualized as isosurfaces of swirling strength $\lambda_{i}$. Here, groups of hairpin vortex structures were observed in most experimental conditions. The center of curvature of the hairpin's head was typically located at $r=1.5 \mathrm{~b}$.

Linear stochastic estimation revealed a hairpin vortex similar to the one observed in the quasi-instantaneous field. A typical spacing between the legs of the hairpin was $0.65 b$, which was independent of the Reynolds number in the range of $1500-5000$.

\section{ACKNOWLEDGMENT}

The authors would like to thank Professor Nao Ninomiya of Utsunomiya University for his helpful discussions.

\section{APPENDIX A: VORTEX IDENTIFICATION}

For vortex identification, we employed the swirling strength $\lambda_{i}$, which is supposed to be a better indicator than vorticity, since the latter necessarily includes the local shearing transformation of fluid while the former does not. We thought the use of $\lambda_{i}$ to be more reliable for a flow such as that of the round jet, which has a thick shear layer, and we were successful in identifying the swirling motion. Figure 13 shows a comparison between the visualizations of vorticity and $\lambda_{i}$. At a glance, both are fairly similar. But, in detail, they are not so similar; for example, in the white dotted circles in Fig. 13, the vortices look warped and sometimes combine when visualized by vorticity.

\section{APPENDIX B: CONVECTIVE VELOCITY}

Throughout this paper, the convective velocity $U_{c}$ was determined to be $U_{c}=\alpha U_{m}$ with $\alpha=0.3$. Although the value of $\alpha$ is reasonable in representing the convective velocity of $r \approx 1.5 b-2.0 b$ according to the profile of $U_{c}$, it is worthwhile to compare the structures visualized using other values of $\alpha$. Figure 14 shows isosurfaces of $\lambda_{i}$ at $x=20 D, \operatorname{Re}=3000$, with the same data and viewpoint as that of Fig. 5, except the $\alpha$ for computing the velocity gradient tensor is different. Note that the axial coordinate $\left(x^{*}=-U_{c} t\right)$ for displaying isosurfaces was calculated using a fixed value of $\alpha=0.3$; otherwise, the structures might be stretched or shrunk by varying the $\alpha$ and comparison would be more difficult. The difference between $\alpha=0.2$ (a) and $\alpha=0.5$ (b) is not obvious and both cases show very similar structures, such as the hairpin, as in the case of $\alpha=0.3$ [shown in Fig. 5(a)]. Some heads of the hairpin vortices are slightly larger for $\alpha=0.2$ than for $\alpha=0.5$, where the contribution of the streamwise gradient of velocity (e.g., $\left.\partial v / \partial x^{*}\right)$ to the $\lambda_{i}$ is more significant than for other parts such as the quasistreamwise hairpin legs, although our conclusion is not affected by this fact.

${ }^{1}$ N. Rajaratnam, Turbulent Jets (Elsevier, New York, 1976).

${ }^{2}$ I. Wygnamski and H. Fiedler, "Some measurements in the self-preserving jet," J. Fluid Mech. 38, 577 (1969).

${ }^{3}$ P. E. Dimotakis, R. C. Miake-Lye, and D. A. Papantoniou, "Structure and dynamics of round turbulent jets," Phys. Fluids 26, 3185 (1983).

${ }^{4}$ S. C. Crow and F. H. Champagne, "Orderly structure in jet turbulence," J. Fluid Mech. 48, 547 (1971).

${ }^{5}$ D. Liepmann and M. Gharib, "The role of streamwise vorticity in the near-field entrainment of round jets," J. Fluid Mech. 245, 643 (1992).

${ }^{6} \mathrm{~S}$. Komori and H. Ueda, "The large-scale coherent structure in the intermittent region of the self-preserving round free jet," J. Fluid Mech. 152, 337 (1985).

${ }^{7} \mathrm{M}$. Yoda, "The instantaneous concentration field in the self-similar region of a high Schmidt number round jet," Ph.D. thesis, Stanford University, 1992.

${ }^{8}$ J. Tso and F. Hussain, "Organized motions in a fully developed turbulent axisymmetric jet," J. Fluid Mech. 203, 425 (1989).

${ }^{9} \mathrm{~N}$. Ninomiya, "The organized structures in an axisymmetric free jet," Ph.D. thesis, Tokyo University, 1992.

${ }^{10}$ H. Suto, K. Matsubara, M. Kobayashi, Y. Hirose, and Y. Matsudaira, "Coherent structures of flow and scalar transfer in a round jet," Proceedings of the 12th International Heat Transfer Conference (Grenoble, France, 2002), Vol. 2, p. 297.

${ }^{11}$ D. P. Hart, "PIV error correction," Exp. Fluids 29, 13 (2000).

${ }^{12}$ M. S. Chong, A. E. Perry, and B. J. Cantwell, "A general classification of three-dimensional flow field," Phys. Fluids A 2, 765 (1990). 
${ }^{13}$ J. Zhou, R. J. Adrian, S. Balachandar, and T. M. Kendall, "Coherent packets of hairpin vortices in channel flow," J. Fluid Mech. 387, 353 (1999).

${ }^{14}$ R. J. Adrian, "Stochastic estimation of conditional structure: a review,"
Appl. Sci. Res. 53, 291 (1994).

${ }^{15}$ T. B. Nickels and I. Marusic, "On the different contributions of coherent structures to the spectra of a turbulent round jet and a turbulent boundary layer," J. Fluid Mech. 448, 367 (2001) 